\title{
Comportement mécanique de sédiments traités par un liant bas carbone type géopolymère et proposition de valorisation matière
}

\author{
Philippe DHERVILLY ${ }^{1}$, Louis JARDIN ${ }^{1}$, Clément BENASSY ${ }^{\mathbf{1}}$, \\ Daniel LEVACHER ${ }^{2}$, William CRUAUD ${ }^{1}$, Valéry LE TURDU ${ }^{3}$
}

1. Neolithe, 5 rue des Ateliers, 49290 Chalonnes-sur-Loire, France.

philippe.dhervilly@neolithe.fr;louis.jardin@neolithe.fr;clement.benassy@neolithe.fr; william.sr.cruaud@neolithe.fr

2. ComUE Normandie Université, Unicaen, M2C UMR 6143 CNRS, 24 rue des Tilleuls, 14000 Caen, France.

daniel.levacher@unicaen.fr

3. Cerema Ouest, site d'Angers, 23 Avenue Amiral Chauvin, Les Ponts-de-Cé, France.

Valery.Le-turdu@cerema.fr

\section{Résumé:}

La valorisation matières des sédiments issu du draguage des ports et voies navigable que ce soit sous forme de granulat ou encore sous forme d'inclusion dans un mortier est une solution à fort impact économique et écologique. En effet cette solution permet à la fois de trouver un exutoire utile aux sédiments mais également de limiter l'extraction de granulats ou autres fillers pour béton et ouvrages de BTP. Cette extraction ayant des conséquences sur les paysages et utilisant une ressource non renouvelable.

Afin de proposer une voie différente de valorisation l'équipe de Neolithe a développé un éco-liant qui forme une matrice composée majoritairement de sédiments floculé non traité. Cette matrice a des propriétés mécaniques, microstructurales et de durabilité suffisante qui permettent d'envisager des applications de valorisation dans le milieu du BTP.

Cet article présente l'étude montrant la faisabilité scientifique d'une telle valorisation de sédiment dans le but d'une application industrielle.

\section{Mots-clés :}

Sédiments, Eco granulat, Traitement et valorisation des sédiments, Digue et ouvrage maritime, BTP

\section{Introduction}

Les sédiments désignent un conglomérat de matériaux, organiques et inorganiques, qui peuvent être emportés par les courants d'eau et le vent. Plusieurs modes de transition des sédiments ont été observés, à savoir : le roulement, la traction, la saltation (suite à des chocs successifs) et la transition par suspension (MOGHRABI, 2018). Des ouvrages tels 


\section{Thème 6 - Gestion durable des zones littorales et estuariennes}

que les barrages, les ports et les ponts peuvent entraver la transition naturelle des sédiments, ce qui réduit la profondeur de navigation des ports et des voies navigables. Ainsi, les opérations de dragage des sédiments sont nécessaires pour maintenir de bonnes conditions de navigabilité des ports et des voies navigables. En France, 31millions de tonnes de sédiments en matière sèche ont été dragués en 2015 (GEODE, 2018). La valorisation de ces sédiments est une alternative à l'immersion en mer et au stockage à terre. Il existe plusieurs voies de valorisation des sédiments : en filière agronomique (épandage agricole) et en génie civil (fabrication des bétons, céramique, production du ciment), (BRGM, 2017). Cependant actuellement la valorisation matière des sédiments utilise majoritairement des liants conventionnels (BANOUNE et al., 2016 ; EL MAHDI SAFHI et al., 2020), souvent à base de clinker qui ont donc un coût écologique élevé et qui nécessite de plus un sédiment peu humide pour pouvoir être utilisé. Ou alors la valorisation implique une étape supplémentaire, la calcination (SAMARA et al., 2009). Parmi les voies potentielles de valorisation, les filières matériaux de remblai, digue et de couche de forme routière figurent parmi celles qui nous semblent les plus intéressantes d'un point de vue économique, environnemental et technique du fait notamment de la proximité des sites de valorisation par rapport aux sites de dragage.

\section{Problématiques et objectifs}

\subsection{Problématiques}

La valorisation des sédiments en génie civil et même en filière agricole requiert au préalable une teneur en eau convenable par rapport à leur mise en œuvre. Les sédiments dragués ont une teneur en eau initiale importante après dragage comprise entre 100 et 600 $\%$, voire plus. Par conséquent, la première étape de la valorisation après le dragage est le séchage. Or, celui- ci constitue une vraie problématique par rapport à la valorisation car un séchage naturel par décantation est relativement lent et peu efficace tandis qu'une déshydratation mécanique ne paraît pas encore adaptée à des volumes importants malgré son efficacité.

La succession de différents modes de transition des sédiments a pour effet de produire des sédiments bruts de propriétés hétérogènes nécessitant par la suite d'adapter le traitement par rapport à une voie de valorisation donnée. Dans sa thèse, ANGER (2014) a développé un outil de pré-orientation quantitatif pour la valorisation de sédiments fins vers la méthode la plus adaptée (intégration dans le béton, dans les matériaux routier et assimilé, en matière céramique ou pour un usage agricole). En complément de cet outil, il nous semble pertinent de proposer une approche qui permet de prédire le comportement du sédiment traité (comportement mécanique ou physico-chimique) en fonction des caractéristiques du sédiment brut et du type de traitement appliqué.

La deuxième étape de la valorisation des sédiments, particulièrement valable pour la construction de routes, remblais et digues, concerne le processus de solidification 


\section{XVİ̀mes Journées Nationales Génie Côtier - Génie Civil \\ Le Havre 2020}

/stabilisation, une approche de traitement bien connue des sols fins par les liants hydrauliques : le ciment et la chaux. Dans le cadre de ce travail, nous étudions le traitement des sédiments en mettant en œuvre des liant bas carbones type géopolymère. Il s'agit de liants obtenus à partir d'un mélange de source(s) aluminosilicate(s) pouvant être issues de sous-produits industriels tels que les cendres volantes et le laitier de haut fourneau, avec des solutions alkali-silicates et ayant montré des propriétés mécaniques et de durabilité intéressante.

Dans notre cas le liant est un liant géopolymère de type alcali-activé. La prise est relativement rapide mais pour optimiser le processus pour tout type d'applications (notamment la fabrication de préfabriqué) nous avons dû accélérer encore cette prise grâce à un traitement thermique limité. Cette méthode nous permet ainsi de prévoir une utilisation de la matière valorisée dans des délais et des coûts acceptable industriellement le tout en ayant un impact carbone limité.

\subsection{Objectif de cette étude}

Il concerne la caractérisation des propriétés microstructurales, mécaniques et de durabilité des sédiments traités en vue de leur utilisation en tant que matériaux de remblai, de sous couche routière et de protection côtière. Les sédiments sont des composés très fins de l'ordre de la dizaine de micromètre. La technologie développée consiste à dissoudre ces sédiments dans une matrice de type cimentaire et ainsi de construire des structures stables à des granulométries plus importantes. Cette technique permet donc de faire des granulats pour sous-couche routière (4-31,5 mm environ) ou encore des blocs de rocher pour fabriquer et/ou consolider des digues. Cette technologie permet également d'aller plus loin et créer des ouvrages moulés de toute forme comme des protections de câbles pour les éoliennes offshore.

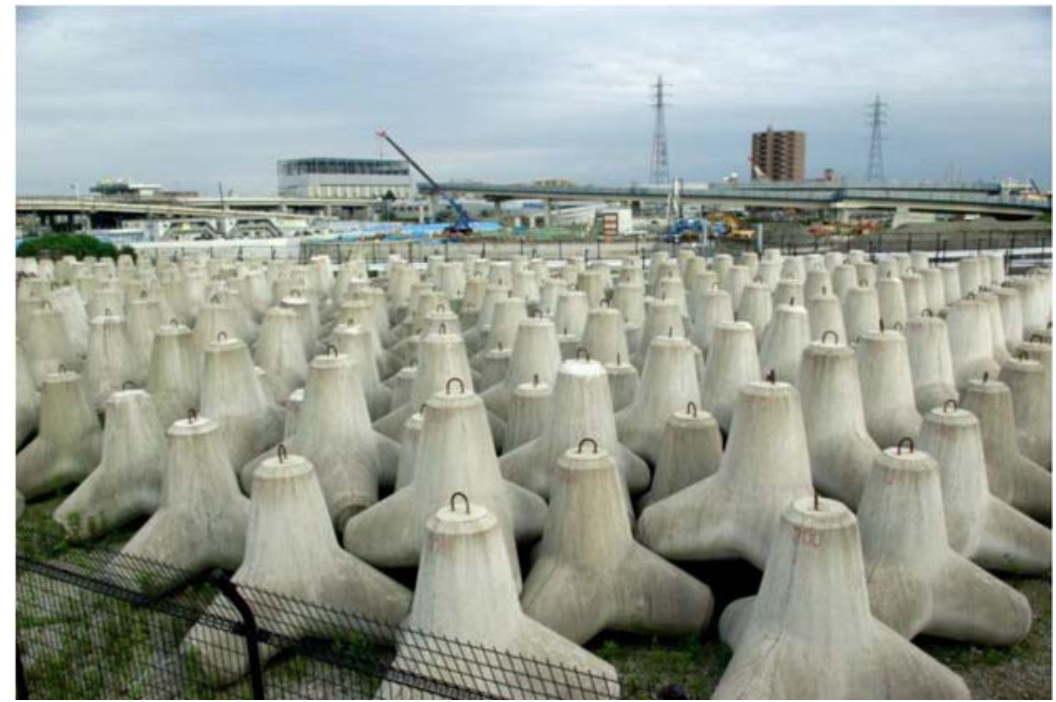

Figure 1. Exemple de tétrapodes préfabriqués avant leur pose, (Wikipédia). 


\section{Thème 6 - Gestion durable des zones littorales et estuariennes}

L'utilisation des tétrapodes est une solution privilégiée dans la consolidation des digues. Leur fabrication à partir de sédiments est envisagée dans le projet, voir figure 1. Afin d'arriver à ce type de matériau, la liaison sédiments-liant alkali activé doit vérifier des propriétés de stabilité chimique et physique ainsi qu'une certaine résistance mécanique. Tout d'abord les sédiments subissent un processus de floculation directement à la sortie du lit du fleuve amenant leur teneur en eau à 30\% de siccité afin d'améliorer le transport de ceux-ci, réduire les effets de l'eau et finalement améliorer leur liaison avec le liant. Ils sont ensuite directement mélangés avec le liant et son activateur sans séchage pendant une dizaine de minute. Après un traitement thermique à basse température d'une heure, le mélange est laissé maturé pendant 28 jours.

\section{Les propriétés microstructurales}

Une des grandes problématiques concerne la liaison entre les fines particules de sédiment et le liant afin de présager de la stabilité physique et chimique de la composition. C'est pour cela qu'une étude microscopique à un âge suffisant ( 7 jours) des échantillons au microscope électronique à balayage en contraste chimique (EVO LS10 ZEISS) a été réalisée afin de juger de l'incorporation du sédiment dans la matrice liante, voir figure 2. Une étude EDX a été menée en parallèle afin d'obtenir des informations sur la répartition chimique de la composition.

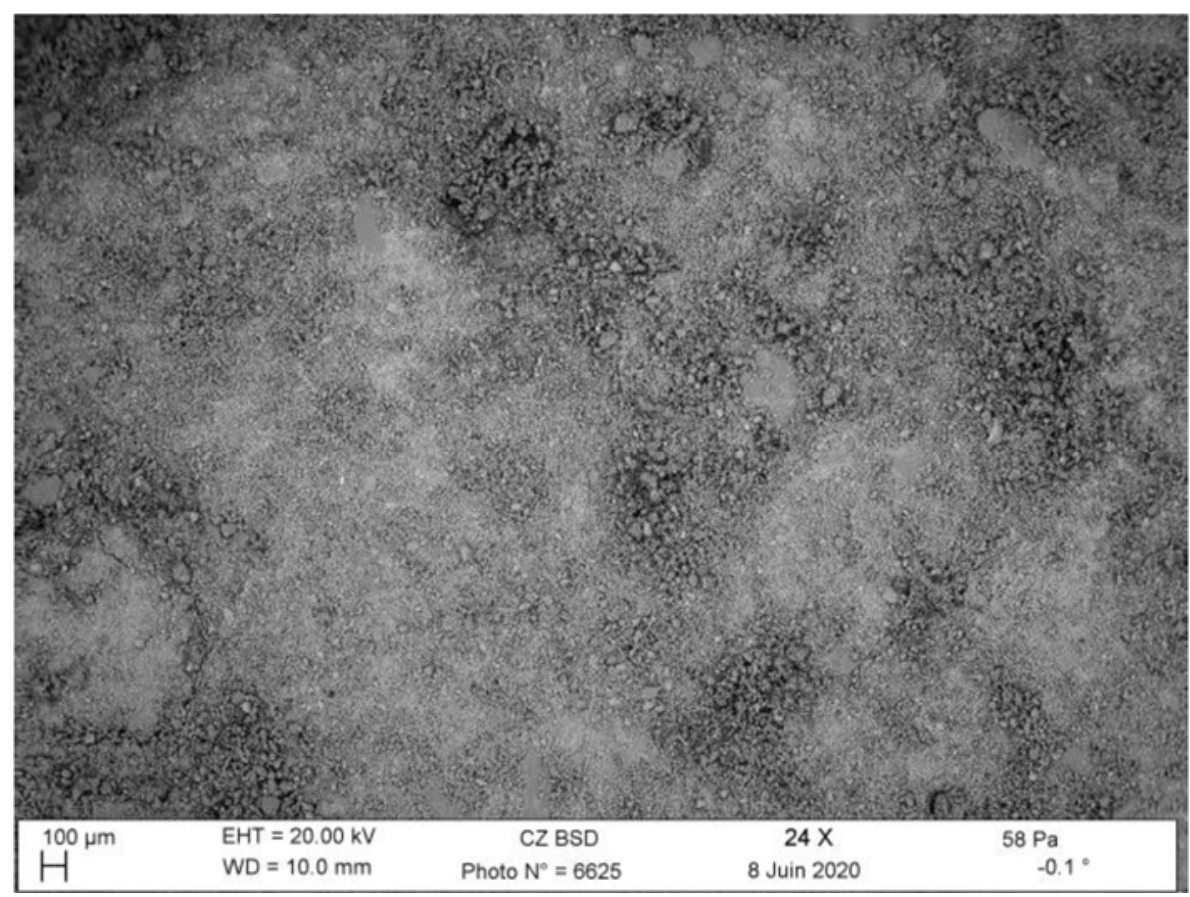

Figure 2. Image MEB de la matrice sédiment-liant au détecteur à contraste chimique, grossissement $x 24$. 


\section{XVI èmes Journées Nationales Génie Côtier - Génie Civil \\ Le Havre 2020}

On observe ainsi grâce à ces outils une structure homogène chimiquement, avec des différences de porosités (phases sombres et phases claires) relativement faibles et uniforme cristallographiquement. De plus la matrice ne comporte quasiment pas de failles et ceci à n'importe quelle échelle. Ceci nous montre donc que les sédiments sont parfaitement intégrés à la matrice de liant ans problème de dispersivité chimique et/ou cristallographique et ceci avec peu de contrainte physique lors de la prise. Ainsi nous avons pu obtenir une intégration fine de l'ordre du micromètre entre les sédiments et le liant, intégration stable chimiquement après la prise et relativement intime.

L'agrandissement nous permet d'observer la capacité de liaison du mélange liantsédiment avec un grain de sable classique, ce qui nous montre que la création d'un type de gel liant-sédiment renforcé au sable est stable et permet donc de moduler la résistance de l'ensemble, voir figure 3.

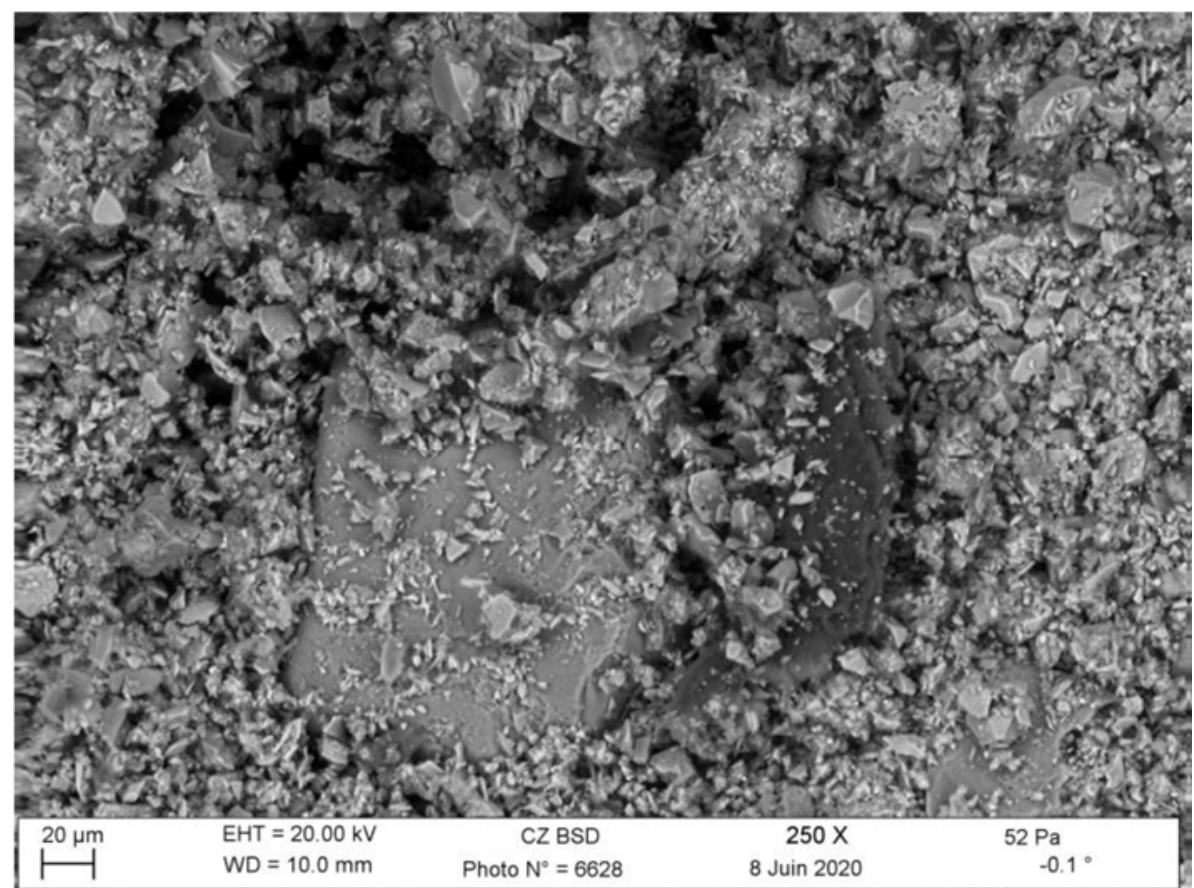

Figure 3.Image MEB d'un grain de sable lié dans la matrice liant-sédiment au détecteur à contraste chimique grossissement $\times 250$.

\section{Les essais mécaniques}

Pour cette partie nous nous sommes inspirés des travaux de thèse de SILITONGA (2010). Les sédiments ont été moulés sous le format $4 \times 4 \times 16$ en suivant la norme NF EN 196-1 relative à la confection de telles éprouvettes pour les applications notamment cimentaires. Un essai de flexion/compression a été fait sur 3 éprouvettes au bout de 7 jours nous donnant une valeur de flexion à 1.1 $\mathrm{MPa}$ et une valeur de compression de $6.8 \mathrm{MPa}$. Une série d'essais à 3-7-14 et 28 jours sera effectuée après la rédaction de cet article. Cette 


\section{Thème 6 - Gestion durable des zones littorales et estuariennes}

valeur ouvre dans tous les cas la possibilité d'utiliser de telles matrices pour de nombreuses applications dans le domaine du BTP.

\section{Les essais de résistance physico-chimiques}

Toujours en inspiration des travaux de la thèse SILITONGA (2010) nous avons fait des tests de mouillage-séchage en alternant $24 \mathrm{~h}$ de mouillage dans une eau à température ambiante puis $24 \mathrm{~h}$ à $60^{\circ} \mathrm{C}$ dans une étuve et nous avons répété ce cycle 5 fois. La matrice donne de bon aspect extérieur après ce cycle augurant de bon résultat malgré l'absence de test de compression post cycle pour l'instant.

\section{Applications envisagées}

Comme nous avons pu le voir, le matériau liant-sédiment est stable physicochimiquement du point de vue microstructural mais également de par sa résistance à l'eau. De plus, les caractéristiques mécaniques sont encourageantes.

Ainsi, au regard des résultats de l'étude, nous pouvons envisager des utilisations variées notamment dans le domaine du BTP mais aussi dans la consolidation de digues ou dans la fabrication de tétrapodes. Nous pourrons donc mener des essais complémentaires afin de tester leur conformité à ces utilisations. L'utilisation en sous couche routière (couche de base et/ou couche de fondation), sous forme de granulat ou encore sous forme de sol stabilisé est également une solution technologiquement faisable qui pourrait permettre de trouver un exutoire suffisamment conséquent pour consommer l'ensemble des sédiments à valoriser. Ces applications diverses pourront être développées individuellement en complément de l'étude générale présentée dans cet article.

\section{Références bibliographiques}

ANGER B. (2014). Caractérisation des sédiments fins des retenues hydroélectriques en vue d'une orientation vers des filières de valorisation matière. Thèse de doctorat, Université de Caen Normandie, 309 p. https://hal-normandie-univ.archives-ouvertes.fr/tel-01938082 BANOUNE B., MELBOUCI B., ROSQUOËT F., LANGLET T. (2016). Treatment of river sediments by hydraulic binders for valorization in road construction. Bulletin of Engineering Geology and the Environment, Vol. 75, pp 1505-1517. https://doi.org/10.1007/s10064-015-0844-4

BRGM (2017). Valorisation à terre des sédiments de dragage : retour d'expérience en France et à l'international, Revue collaborative, 117 p. http://infoterre.brgm.fr/rapports/RP-67329FR.pdf

EL MAHDI SAFHI A., RIVARD P., YAHIA A., BENZERZOUR M., KHAYAT K.H. (2020). Valorization of dredged sediments in self-consolidating concrete: Fresh, hardened, and microstructural properties. Journal of Cleaner Production, Vol. 263, 121472, https://doi.org/10.1016/j.jclepro.2020.121472 


\section{XVİ̀mes Journées Nationales Génie Côtier - Génie Civil \\ Le Havre 2020}

GEODE (2018). Dragages et immersions en mer et en estuaire, Revue collaborative, 90 p. https://www.cerema.fr/system/files/documents/2018/04/GEODE_BPE_revue_0702018_VF.pdf

MOGHRABI I. (2018). Modélisation du comportement mécanique des sédiments traités et étude d'une nouvelle voie de leur valorisation par des géopolymères. Thèse de doctorat, Université de Bretagne-Loire, 263 p.

SAMARA M., LAFHAJ Z., CHAPISEAU C. (2009). Valorization of stabilized river sediments in fired clay bricks: Factory scale experiment. Journal of Hazardous Materials, Vol. 163(2-3), pp 701-710, https://doi.org/10.1016/j.jhazmat.2008.07.153

SILITONGA E. (2010). Valorisation des sédiments marins contaminés par solidification/ stabilisation à base de liants hydrauliques et de fumée de silice. Thèse de doctorat, Université de Caen Normandie, 245 p. https://tel.archives-ouvertes.fr/tel-00705226 
Thème 6 - Gestion durable des zones littorales et estuariennes 